\title{
EDITORIAL
}

\section{Inflammation, Thrombosis and Vascular Biology: Translating Ideas into Cardiovascular Research and Therapy}

Advances in basic and clinical sciences over the past decades have substantially improved our understanding of inflammatory and thrombotic pathways implicated in atherogenesis. It is now clear that multiple genetic and environmental factors interfere with the natural course of atherosclerosis in early life and lead toward premature clinical manifestations in the adolescence and in the middle age. Importantly, various molecular and cellular agents with pro-atherogenic properties have been identified, and similarities have been found between atherosclerotic vascular disease, chronic inflammatory disorders and cancer [1, 2], all of which can be viewed as diseases of premature aging. Identification of common pathways of aging, and premature morbidity and mortality in these disorders has prompted the search for therapeutic means with universal beneficial effects. In fact, over the past decade, we have witnessed a strong emphasis on anti-inflammatory effects of cardiovascular drugs, such as angiotensin converting enzyme inhibitors (ACE-I), statins, aspirin, which are believed to reduce incidence of vascular events by acting not only on their primary therapeutic targets but also on the inflammatory process [3].

The first report on predictive vascular value of levels of C-reactive protein (CRP) [4] revolutionized cardiovascular research, and directed efforts of cardiologists and allied professionals toward elaboration of a new cardiovascular preventive strategy based on the current view on atherosclerosis as an inflammatory disorder.

Preventive measures against established cardiovascular risk factors (e.g., dyslipidemia, hypertension, active and passive smoking, obesity) proved efficient in reducing inflammatory burden in the general population and in patients with established cardiovascular disease, and it seems obvious that simple non-medicamentous preventive measures started early in life may substantially reduce incidence of cardiovascular events and contribute to healthy aging [5].

As more data on genetic polymorphisms underlying inflammation have accumulated [6], it has become possible to outline directions of cardiovascular prevention, which should take into account variability of expression of major mediators of systemic inflammation (e.g., CRP, pro-inflammatory cytokines). Whether the screening for genetic polymorphisms of CRP and other mediators should be part of community-based cardiovascular preventive measures is still not clear. Added to the complexity, the number of inflammatory mediators of atherogenesis is constantly increasing [7], and it is still poorly understood how genetic polymorphisms of so many 'major' and 'minor' contributors to atherogenesis interact with each other and form basis for accelerated atherosclerosis. Perhaps future basic research on animal models of atherosclerosis with knock-out inflammatory genes will provide more specific answers. In fact, the latter experimental approach proved useful in understanding a variety of molecular mechanisms implicated in hypertension, atherosclerosis and other cardiovascular and hematological disorders [8].

An important factor, which is closely but not exclusively related to the genetic predisposition to accelerated atherosclerosis, is ethnicity. It is well known that the majority of recent large trials on cardiovascular disease prevention and treatment are based on cohorts of subjects from Western countries, mainly Caucasians. However, ethnic origin, geographic location, level of education, social, religious, and cultural factors may substantially influence the course of cardiovascular disease. Over the past few years, importance of these factors has been appreciated in a few well-designed studies comparing cardiovascular risk factors in cohorts of subjects from the Eastern and Western countries [9]. Researchers' interest toward an excess of cardiovascular morbidity and mortality among South Asians is growing in the UK [10] and the US [11]. Recently, several new markers of increased cardiovascular risk in South Asians have been proposed, with dysfunctional high density lipoproteins and high sensitivity CRP being widely studied [12]. Importantly, the loss of anti-inflammatory properties of HDL was associated with an increased atherosclerotic burden in South Asians [13]. The obtained data indicate the need for large scale prospective studies, which will allow elaborating ethnic specific cardiovascular prevention guidelines [14].

The concept of atherosclerosis as an inflammatory disorder has been substantially enriched due to large cohort studies on enhanced atherosclerotic cardiovascular disease in high-grade inflammatory disorders such as systemic lupus erythematosus (SLE) and rheumatoid arthritis (RA) [15, 16]. Both diseases are now viewed as clinical models of inflammation-induced atherogenesis. Anti-rheumatic drugs used in these disorders are believed to suppress intensity of inflammatory and prothrombotic pathways of atherogenesis and, thus, to prevent vascular events. Specifically designed prospective studies to prove this hypothesis are warranted. One of the relatively safe anti-rheumatic drugs, methotrexate, has been already suggested for the suppression of overproduction of CRP and prevention of cardiovascular events in the general population [17]. It is expected that elaboration of other clinical models of atherogenesis, particularly those associated with low-grade systemic inflammation, will further expand the armamentarium of potent anti-inflammatory drugs useful in cardiovascular disease states [18]. A few recent studies on non-rheumatic cardiovascular disease have already outlined relatively safe anti-inflammatory profile of colchicine, which was initially demonstrated its efficiency in familial Mediterranean fever (FMF), an emerging clinical model of atherosclerosis associated with low-grade inflammation [19].

Interestingly, not all systemic inflammatory disorders predispose to accelerated atherogenesis. Wegener's granulomatosis (WG) [20], Behçet's disease (BD) [21], and some other vasculitides are good examples of clinical course of chronic inflammation, damaging the endothelium, and leading to vascular aneurysms, arterial and venous thrombosis but not to atherosclerotic 
vascular disease. Mechanisms of this vasculitic damage, shifting inflammatory burden toward pro-thrombotic state, are not fully understood. Perhaps future large studies on genetic polymorphisms responsible for overexpession of thrombogenic factors in $\mathrm{BD}$ and other vasculitides will partially explain this phenomenon [22].

It is also worth noting that specifically designed comparative studies on cardiovascular manifestations and atherogenesis in different low- (FMF, BD) and high-grade inflammatory disorders (RA, SLE) will make it possible to better understand atherogenic and pro-thrombotic implications of inflammation, and to provide the clinician with recommendations for antithrombotic therapy. Unfortunately, currently available guidelines on antithrombotic therapy do not provide answers to the questions of how and when to treat patients with inflammatory disorders at high atherothrombotic risk. To some extent, it is due to high risk of bleeding conferred by anti-platelet and anti-coagulant therapies in chronic inflammatory disorders, and due to exclusion of patients with these disorders from the major trials. Of note, patients with $\mathrm{BD}$, who frequently present with pulmonary aneurysms, are at high risk of pulmonary hemorrhage, which may further increase in the case of anticoagulation. These and related issues of antithrombotic therapy are comprehensively covered in some reviews of this issue [22, 23].

Intensity of inflammation is a crucial pathophysiological factor, which should be viewed as a target of current anti-rheumatic and anti-thrombotic therapies. However, each inflammatory disorder has its specific targets, which specifically contribute to vascular pathology (e.g., neutrophilic aggression in FMF, BD, WG, autoantibodies in SLE and RA). In each of these disorders, vasculopathy is established in numerous observational studies with healthy controls [24]. What is more interesting and useful is to compare the contribution of these specific factors on vasculopathy in comparative studies with appropriately matched inflammatory disease controls.

Therapeutic targets in inflammatory disorders widely vary. In RA, for example, physical inactivity can be viewed as a specific target. Enhanced physical activity is thought to decrease inflammatory burden and ameliorate endothelial function in this disorder [25]. Mechanisms of endothelial dysfunction in RA and targeted action of physical activity on inflammatory changes in the endothelium are analyzed in this issue [25]. It is hoped that future studies of the effects of physical activity on endothelial, inflammatory and pro-thrombotic agents in RA will have practical implications for patients with inflammatory polyarthritis and non-RA subjects with sedentary lifestyle.

Finally, over the past decade, substantial achievements were made in the field of stem cell research and therapy. It was shown that chronic inflammation interferes with the process of endothelial regeneration and, thereby, contributes to endothelial dysfunction [26]. Attempts were made to use bone marrow derived stem cells for therapeutic purposes in several cardiovascular disorders [27]. Whether this approach will be also useful in chronic inflammatory disorders is not clear. Perhaps the most important limiting factor, in this regard, is the difficulty to isolate stem/progenitor cells with the required cardiovascular or endothelial differentiation properties [28].

Selected reviews presented in this issue summarize available data on inflammation-induced atherosclerosis, thrombosis and vascular biology. In some of these reviews, special attention is paid to the ongoing attempts to elaborate clinical models of accelerated atherosclerosis associated with low- and high-grade inflammatory disorders, which shed light into the targets for future cardiovascular preventive measures. It is evident that multiple genetic and environmental factors underlie activation of pro-atherogenic molecular and cellular agents. Atherosclerosis shares pathophysiological features with a variety of chronic inflammatory disorders and cancer, and better understanding of vasculopathy in each of these conditions will boost research studies for years to come, and will lead to practical recommendations for healthy subjects and patients with established cardiovascular disease.

\section{ACKNOWLEDGEMENTS}

I would like to extend my gratitude to all those who have given their much valuable time and efforts and contributed to this special issue as authors, reviewers and editors. Special thanks to all those who thoroughly reviewed, edited, and provided their opinion on the all manuscripts of this special issue: Cocco G, La Regina M, Kelishadi R, Cicero AFG, Yildiz M, Westerweel PE, Yüksel Ş, Assari S, Dotsenko O, Hristova K, Garelnabi M, Margariti A, Habi O, Syed M.

\section{REFERENCES}

[1] McNicol A, Israels SJ. Beyond hemostasis: the role of platelets in inflammation, malignancy and infection. Cardiovasc Hematol Disord Drug Targets 2008; 8: 99-117.

[2] Filippatos TD, Liberopoulos EN, Pavlidis N, Elisaf MS, Mikhailidis DP. Effects of hormonal treatment on lipids in patients with cancer. Cancer Treat Rev 2009; 35: 175-84.

[3] Gasparyan AY, Watson T, Lip GY. The role of aspirin in cardiovascular prevention: implications of aspirin resistance. J Am Coll Cardiol 2008; 51: 1829-43.

[4] Ridker PM, Cushman M, Stampfer MJ, Tracy RP, Hennekens CH. Inflammation, aspirin, and the risk of cardiovascular disease in apparently healthy men. N Engl J Med 1997; 336: 973-9.

[5] Kelishadi R. Inflammation-induced atherosclerosis as a target for prevention of cardiovascular diseases from early life. Open Cardiovasc Med J 2010; 4: 24-9.

[6] Shanker J, Kakkar VV. Implications of genetic polymorphisms in inflammation-induced atherosclerosis. Open Cardiovasc Med J 2010; 4: 30-7.

[7] Abbasi SH, Boroumand MA. Expanded network of inflammatory markers of atherogenesis: where are we now? Open Cardiovasc Med J 2010; 4: 3844.

[8] Ugurlucan M, Gasparyan AY, Darban H. Nobel Prize in physiology or medicine for the year 2007: breakthrough in pathophysiology and experimental therapy of cardiovascular and other diseases. J Teh Univ Heart Ctr 2008; 3: 233-4. 
[9] Kelishadi R, Schwandt P, Haas G-M, Hosseini M, Mirmoghtadaee P. Reference curves of anthropometric indices and serum lipid profiles in representative samples of Asian and European children. Arch Med Sci 2008; 4: 329-35.

[10] Lip GY, Barnett AH, Bradbury A, et al. Ethnicity and cardiovascular disease prevention in the United Kingdom: a practical approach to management J Hum Hypertens 2007; 21: 183-211.

[11] Ivey SL, Mehta KM, Fyr CL, Kanaya AM. Prevalence and correlates of cardiovascular risk factors in South Asians: population-based data from two California surveys. Ethn Dis 2006; 16: 886-93.

[12] Dodani S. Excess coronary artery disease risk in South Asian immigrants: can dysfunctional high-density lipoprotein explain increased risk? Vasc Health Risk Manag 2008; 4: 953-61.

[13] Dodani S, Marion L, Sharma G, Reed GL, George V. The association of carotid intima media thickness with dysfunctional HDL in South Asians. Arch Med Sci 2008; 4: 40-6.

[14] Dodani S. Atherothrombosis in South Asians: Implications of Atherosclerotic and Inflammatory Markers. Open Cardiovasc Med J 2010; 4: 45-50.

[15] Nikpour M, Urowitz MB, Gladman DD. Epidemiology of atherosclerosis in systemic lupus erythematosus. Curr Rheumatol Rep 2009; 11: 248-54.

[16] Gabriel SE, Michaud K. Epidemiological studies in incidence, prevalence, mortality, and comorbidity of the rheumatic diseases. Arthritis Res Ther 2009; 11: 229.

[17] Ridker PM. Testing the inflammatory hypothesis of atherothrombosis: scientific rationale for the cardiovascular inflammation reduction trial (CIRT). J Thromb Haemost 2009; 7 (Suppl 1): 332-9.

[18] Gasparyan AY, Ugurlucan M. The emerging issue of cardiovascular involvement in familial Mediterranean fever. Arch Med Sci 2008; 4: 465-7.

[19] Yüksel Ş, Ayvazyan L, Gasparyan AY. Familial Mediterranean fever as an emerging clinical model of atherogenesis associated with low-grade inflammation. Open Cardiovasc Med J 2010; 4: 51-6.

[20] Cocco G, Gasparyan AY. Myocardial ischemia in Wegener's granulomatosis: coronary atherosclerosis versus vasculitis. Open Cardiovasc Med J 2010; 4: 57-62.

[21] Cocco G, Gasparyan AY. Behçet's disease: an insight from a cardiologist's point of view. Open Cardiovasc Med J 2010; 4: 63-70.

[22] La Regina M, Gasparyan AY, Orlandini F, Prisco D. Behçet's disease as a model of venous thrombosis. Open Cardiovasc Med J 2010; 4: 71-7.

[23] Karimi M, Cohan N. Cancer-associated thrombosis. Open Cardiovasc Med J 2010; 4: 78-82.

[24] Yildiz M. Arterial distensibility in chronic inflammatory rheumatic disorders. Open Cardiovasc Med J 2010; 4: 83-8.

[25] Metsios GS, Stavropoulos-Kalinoglou A, Sandoo A, et al. vascular function and inflammation in rheumatoid arthritis: the role of physical activity. Open Cardiovasc Med J 2010; 4: 89-96.

[26] Westerweel PE, Verhaar MC. Endothelial progenitor cell dysfunction in rheumatic disease. Nat Rev Rheumatol 2009; 5: 332-40.

[27] Dotsenko O, Jahangiri M. Endogenous stem cells in patients undergoing coronary artery bypass graft surgery. Eur J Cardiothorac Surg 2009; 36: 56371 .

[28] [28] Dotsenko O. Stem/progenitor cells, atherosclerosis and cardiovascular regeneration. Open Cardiovasc Med J 2010; 4: $97: 104$.

Armen Yuri Gasparyan

(Guest Editor)

Dudley Group of Hospitals NHS Foundation Trust,

Dudley, West Midlands, UK

E-mails: armen.gasparyan@dgoh.nhs.uk 


\title{
Meet the Guest Editor
}

\author{
Armen Yuri Gasparyan \\ (Guest Editor) \\ Dudley Group of Hospitals NHS Foundation Trust, \\ Dudley, West Midlands, UK \\ E-mails: armen.gasparyan@dgoh.nhs.uk \\ a.gasparyan@gmail.com
}

Dr. Armen Yuri Gasparyan is a graduate of the Yerevan Medical University (1990-1997) and clinical residency in cardiology (1997-2000). He received his PhD degree for studies of cardiovascular involvement in familial Mediterranean fever (20002003). He passed clinical internship in internal medicine at the Barmherzigen Bruder General Hospital, Graz, Austria (2002, 2008). For numerous publications, training of English-speaking medical students and clinical duties, he was awarded an academic title of associate professor of medicine (2006). In 2004-2006, Dr. Gasparyan was serving as a consultant in clinical cardiology and rheumatology at the Unit of Internal Medicine, Central Clinical Military Hospital, Yerevan, Armenia. He has been granted postdoctoral fellowship at the Haemostasis, Thrombosis and Vascular Biology Unit, University Department of Medicine, City Hospital, Birmingham, UK (2007-2008), and at the Clinical Research Unit, Dudley Group of Hospitals NHS Foundation Trust, West Midlands, UK (since 2008).

He is an active member of editorial boards of The Open Cardiovascular Medicine Journal, Rheumatology International, Current Drug Therapy, Inflammation and Allergy ^ Drug Targets, and American Journal of Biomedical Sciences, and was awarded membership of the World Association of Medical Editors (since 2008) and European Association of Science Editors (since 2009).

He authored more than 80 publications. His latest papers were published in Journal of the American College of Cardiology and Current Vascular Pharmacology. 